\title{
SIMPLE VIRASORO MODULES INDUCED FROM CODIMENSION ONE SUBALGEBRAS OF THE POSITIVE PART
}

\author{
VOLODYMYR MAZORCHUK AND EMILIE WIESNER \\ (Communicated by Kailash C. Misra)
}

Abstract. We construct a new five-parameter family of simple modules over the Virasoro algebra.

\section{INTRODUCTION AND DESCRIPTION OF THE RESULTS}

We denote by $\mathbb{N}$ the set of positive integers and by $\mathbb{Z}_{+}$the set of all nonnegative integers. For a Lie algebra $\mathfrak{a}$ we denote by $U(\mathfrak{a})$ the universal enveloping algebra of a.

Let $\mathfrak{V}$ denote the complex Virasoro algebra, that is the Lie algebra with basis $\left\{\mathrm{c}, 1_{i}: i \in \mathbb{Z}\right\}$ and the Lie bracket defined (for $i, j \in \mathbb{Z}$ ) as follows:

$$
\left[1_{i}, 1_{j}\right]=(j-i) 1_{i+j}+\delta_{i,-j} \frac{i^{3}-i}{12} \mathrm{c} ; \quad\left[1_{i}, \mathrm{c}\right]=0 .
$$

This algebra plays an important role in various questions of mathematical physics; see $\underline{\mathrm{KR}}$.

Classical classes of simple weight $\mathfrak{V}$-modules are simple highest weight modules (see $[\mathrm{FF}$ ) and intermediate series modules. Put together, these two classes exhaust all simple weight $\mathfrak{V}$-modules with finite-dimensional weight spaces (see $\mathrm{Mt}$ ) and even those containing a nonzero finite-dimensional weight space (see MZ1]). We also refer the reader to the recent monograph [IK] for a detailed survey of the classical part of the representation theory of $\mathfrak{V}$. There are a number of other examples of simple $\mathfrak{V}$-modules constructed in [CM, Zh, OW, GLZ, LZ, LGZ, MZ2 using various tricks. The present paper contributes with a new trick leading to a new family of examples depending on five parameters.

For a nonzero $z \in \mathbb{C}$, denote by $\mathfrak{a}_{z}$ the linear span of $1_{k}-z^{k-1} 1_{1}$, where $k \geq 2$, in $\mathfrak{V}$. It is easy to check that $\mathfrak{a}_{z}$ is in fact a Lie subalgebra in $\mathfrak{V}$. For a fixed $\mathbf{m}:=\left(m_{2}, m_{3}, m_{4}\right) \in \mathbb{C}^{3}$, define an $\mathfrak{a}_{z}$-action on $\mathbb{C}$ by

- $\left(1_{i}-z^{i-1} 1_{1}\right) \cdot 1=m_{i}$ for $i=2,3,4$;

- $\left(\mathrm{l}_{i}-z^{i-1} \mathfrak{l}_{1}\right) \cdot 1=-(i-4) m_{3} z^{i-3}+(i-3) m_{4} z^{i-4}$ for $i>4$.

It's straightforward to verify that this gives an $\mathfrak{a}_{z}$-module. We denote it by $\mathbb{C}_{\mathbf{m}}$.

For a fixed $\theta \in \mathbb{C}$ consider the $\mathfrak{V}$-module

$$
\operatorname{Ind}_{z, \theta}\left(\mathbb{C}_{\mathbf{m}}\right):=U(\mathfrak{V}) \otimes_{U\left(\mathfrak{a}_{z}\right)} \mathbb{C}_{\mathbf{m}} /(\mathbf{c}-\theta) U(\mathfrak{V}) \otimes_{U\left(\mathfrak{a}_{z}\right)} \mathbb{C}_{\mathbf{m}} .
$$

Our main result is the following claim.

Received by the editors September 18, 2012 and, in revised form, November 17, 2012. 2010 Mathematics Subject Classification. Primary 17B10, 17B20, 17B65, 17B66, 17 B68. 
Theorem 1. Let $\mathbf{m} \in \mathbb{C}^{3}$ and $z \in \mathbb{C} \backslash\{0\}$ be such that

$$
z m_{3} \neq m_{4}, 2 z m_{2} \neq m_{3}, 3 z m_{3} \neq 2 m_{4} \text {, and } z^{2} m_{2}+m_{4} \neq 2 z m_{3} .
$$

Then for any $\theta \in \mathbb{C}$ the $\mathfrak{V}$-module $\operatorname{Ind}_{z, \theta}\left(\mathbb{C}_{\mathbf{m}}\right)$ is simple.

We start the paper by characterizing $\mathfrak{a}_{z}$ as a special family of codimension one subalgebras in the positive part $\mathfrak{n}$ of $\mathfrak{V}$ in Section 2, Our proof of Theorem 1 consists of three steps: we first induce $\mathbb{C}_{\mathfrak{m}}$ up to $\mathfrak{n}$ in Section 3 , then we study the induction of $\mathbb{C}_{\mathbf{m}}$ to the Borel subalgebra of $\mathfrak{V}$ in Section 4 and, finally, we complete the proof in Section 5 .

\section{Subalgebras in $\mathfrak{n}$ of COdimension one}

Denote by $\mathfrak{n}$ the "positive part" of $\mathfrak{V}$, that is, the Lie subalgebra spanned by $\mathfrak{l}_{i}$, where $i \geq 1$. In this section we characterize $\mathfrak{a}_{z}$ as a special family of codimension one subalgebras in $\mathfrak{n}$. We start with the following lemma.

Lemma 2. Let $\mathfrak{x} \subseteq \mathfrak{n}$ be a Lie subalgebra of codimension one.

(a) If $1_{1} \in \mathfrak{x}$, then $\mathfrak{1}_{k}, 1_{k+1}, \ldots \in \mathfrak{x}$ for some $k \in \mathbb{N}$.

(b) If $1_{s} \in \mathfrak{x}$ for some $s \in \mathbb{N}$, then $\mathfrak{l}_{i s}, 1_{(i+1) s}, 1_{(i+2) s}, \ldots \in \mathfrak{x}$ for some $i \in \mathbb{N}$.

(c) If $1_{i s}, 1_{(i+1) s}, 1_{(i+2) s}, \ldots \in \mathfrak{x}$ for some $s, i \in \mathbb{N}$, then $1_{k}, 1_{k+1}, \ldots \in \mathfrak{x}$ for some $k$.

Proof. We start with claim (a) . If $I_{k} \in \mathfrak{a}$ for some $k>1$, then claim (国) follows immediately. Therefore, assume that this is not the case and in particular that claim (国) is false. Since $\mathfrak{x}$ has codimension 1 and $\mathfrak{l}_{k} \notin \mathfrak{x}$ for any $k>1$, we must have that $1_{k}-a_{k} 1_{k+1} \in \mathfrak{x}$ for all $k \geq 2$ and some $0 \neq a_{k} \in \mathbb{C}$. Also, $\left[1_{1}, 1_{k}-a_{k} 1_{k+1}\right] \in \mathfrak{x}$, which implies $a_{k+1}=\frac{k}{k-1} a_{k}=k a_{2}$. On the other hand,

$$
\begin{aligned}
{\left[1_{2}-a_{2} l_{3}, l_{3}-a_{3} l_{4}\right] } & =l_{5}-2 a_{3} l_{6}+a_{2} a_{3} l_{7} \\
& =l_{5}-4 a_{2} l_{6}+2 a_{2}^{2} l_{7} .
\end{aligned}
$$

Since $1_{5}-a_{5} l_{6}=1_{5}-4 a_{2} l_{6}$, this implies that $1_{7} \in \mathfrak{x}$, a contradiction. This proves claim (a). Claim (b) follows from claim (a) as the span of all $1_{j s}, j \in \mathbb{N}$, is isomorphic to $\mathfrak{n}$ as a Lie algebra.

To prove claim (IC) we show that for any $1 \leq p<s$ there is $j \in \mathbb{Z}_{+}$such that $\mathfrak{l}_{p+j s} \in \mathfrak{x}$. Assume that this is not the case. Then $\mathfrak{l}_{p+j s}-a_{j} 1_{p+(j+1) s} \in \mathfrak{x}$ for all $j \in \mathbb{Z}_{+}$and some $0 \neq a_{j} \in \mathbb{C}$. Also, $\left[1_{i s}, 1_{p+j s}-a_{j} 1_{p+(j+1) s}\right] \in \mathfrak{x}$, which implies $a_{i+j}=\frac{p+(j+1-i) s}{p+(j-i) s} a_{j}$. Hence $a_{2 i+j}=\frac{p+(j+1-i) s}{p+(j-i) s} \frac{p+(j+1) s}{p+j s} a_{j}$.

On the other hand, $\left[1_{2 i s}, 1_{p+j s}-a_{j} 1_{p+(j+1) s}\right] \in \mathfrak{x}$, which implies

$$
a_{2 i+j}=\frac{p+(j+1-2 i) s}{p+(j-2 i) s} a_{j} .
$$

This gives

$$
\frac{p+(j+1-i) s}{p+(j-i) s} \cdot \frac{p+(j+1) s}{p+j s}=\frac{p+(j+1-2 i) s}{p+(j-2 i) s}
$$

for all $j \in \mathbb{Z}_{+}$. This means that the following multisets (the negatives of the roots with respect to the variable $j s$ ) coincide:

$$
\{(1-i) s+p, p+s, p-2 i s\}=\{p-i s, p, p+(1-2 i) s\},
$$

and thus

$$
\{(1-i) s, s,-2 i s\}=\{-i s, 0,(1-2 i) s\}
$$


Since both $i, s \in \mathbb{N}$, the only way to have a zero on the left hand side is to have $i=1$, which implies

$$
\{0, s,-2 s\}=\{-s, 0,-s\} .
$$

Now the left hand side contains a positive integer, while the right hand side does not. This is a contradiction which proves claim (ㄷ) .

Proposition 3. Suppose $\mathfrak{x} \subseteq \mathfrak{n}$ is a subalgebra of codimension one and there is no $k$ such that $\mathfrak{1}_{k}, 1_{k+1}, \ldots \in \mathfrak{x}$. Then $\mathfrak{x}$ has a basis of the form $\left\{\mathfrak{1}_{k}-z^{k-1} 1_{1} \mid k \geq 2\right\}$ for some nonzero $z \in \mathbb{C}$.

Proof. By Lemma 2 we have $1_{s} \notin \mathfrak{x}$ for any $s \in \mathbb{N}$. As $\mathfrak{x}$ has codimension one in $\mathfrak{n}$, it must have a basis of the form $\left\{1_{k}-a_{k} 1_{1} \mid k \geq 2\right\}$ for some nonzero $a_{k} \in \mathbb{C}$.

For $2 \leq i<j$ consider

$$
\begin{aligned}
{\left[\mathrm{l}_{i}+\right.} & \left.a_{i} \mathrm{l}_{1}, \mathrm{l}_{j}+a_{j} \mathrm{l}_{1}\right] \\
= & (j-i) \mathrm{l}_{i+j}-(j-1) a_{i} \mathrm{l}_{j+1}+(i-1) a_{j} \mathrm{l}_{i+1} \\
= & (j-i)\left(\mathrm{l}_{i+j}-a_{i+j} \mathrm{l}_{1}\right)-(j-1) a_{i}\left(\mathrm{l}_{j+1}-a_{j+1} \mathrm{l}_{1}\right) \\
& +(i-1) a_{j}\left(\mathrm{l}_{i+1}-a_{i+1} \mathrm{l}_{1}\right) \\
& +\left((j-i) a_{i+j}-(j-1) a_{i} a_{j+1}+(i-1) a_{i+1} a_{j}\right) \mathrm{l}_{1} .
\end{aligned}
$$

The condition $\left[\mathfrak{l}_{i}+a_{i} \mathbf{l}_{1}, \mathfrak{l}_{j}+a_{j} \mathfrak{l}_{1}\right] \in \mathfrak{x}$ implies

$$
D_{i, j}:=(j-i) a_{i+j}-(j-1) a_{i} a_{j+1}+(i-1) a_{i+1} a_{j}=0 .
$$

Taking $i=2$ and $j=3,4, \ldots$ we get a recursive formula which uniquely determines $a_{5}, a_{6}, \ldots$ in terms of $a_{2}, a_{3}, a_{4}$.

Let $I$ be the ideal in $\mathbb{C}\left[a_{2}, a_{3}, a_{4}, a_{5}, a_{6}, a_{7}, a_{8}, a_{9}\right]$ generated by $D_{2,3}, D_{2,4}, D_{2,5}$, $D_{2,6}, D_{2,7}, D_{3,4}, D_{3,5}, D_{3,6}$ and $D_{4,5}$. Computing the Gröbner basis of $I$ with respect to the lexicographic order for which

$$
a_{9}>a_{8}>a_{7}>a_{6}>a_{5}>a_{4}>a_{3}>a_{2},
$$

we get that $I$ contains $a_{3}^{6}-a_{3}^{5} a_{2}^{2}$, which implies $a_{3}=a_{2}^{2}$ since $a_{3} \neq 0$. Computing the Gröbner basis of $I$ with respect to the lexicographic order for which

$$
a_{9}>a_{8}>a_{7}>a_{6}>a_{5}>a_{2}>a_{3}>a_{4},
$$

we get that $I$ contains $a_{4}^{3} a_{2}-a_{4}^{2} a_{3}^{2}$. Using $a_{3}=a_{2}^{2}$ and $a_{4}, a_{2} \neq 0$, we get $a_{4}=a_{2}^{3}$. This means that all $a_{k}$ are uniquely determined by the value of $a_{2}$.

At the same time, it is easy to check that $a_{k}=a_{2}^{k-1}$ satisfies (2.1) and hence defines a subalgebra.

For a nonzero $z \in \mathbb{C}$ we denote by $\mathfrak{a}_{z}$ the subalgebra constructed in Proposition 3. This one-parameter family of subalgebras exhausts all codimension one subalgebras of $\mathfrak{n}$ which do not contain $\mathfrak{l}_{k}, \mathfrak{l}_{k+1}, \ldots$ for some $k$. Modules induced from subalgebras of $\mathfrak{n}$ containing $\mathfrak{l}_{k}, \mathfrak{1}_{k+1}, \ldots$ for some $k$ were studied in [MZ2]. All such modules fit into the general Whittaker setup for $\mathfrak{V}$ defined in [BM]. Simple modules which are induced from simple 1-dimensional $\mathfrak{a}_{z}$-modules do not fit into this general Whittaker setup. They are the objects of our study in the present paper. 


\section{INDUCTION TO $\mathfrak{n}$}

For $\mathbf{m}:=\left(m_{1}, m_{2}, m_{3}\right) \in \mathbb{C}^{3}$ define $V_{\mathbf{m}}=\operatorname{Ind}_{\mathfrak{a}_{z}}^{\mathfrak{n}}\left(\mathbb{C}_{\mathbf{m}}\right)$. Since $\mathfrak{n}$ has a basis $\mathfrak{l}_{1}, \mathfrak{l}_{2}-z 1_{1}, \mathfrak{l}_{3}-z^{2} \mathfrak{l}_{1}, \ldots$, the PBW Theorem implies that $V_{\mathbf{m}}$ has a basis $\left\{\mathbf{l}_{1}^{k} \otimes 1 \mid\right.$ $k \geq 0\}$.

Proposition 4. The $\mathfrak{n}$-module $V_{\mathbf{m}}$ is simple if and only if am $m_{3} \neq m_{4}$.

Proof. Suppose $0 \neq M \subseteq V_{\mathbf{m}}$. Since $l_{1}$ acts freely on $M$, it follows that $V_{\mathbf{m}} / M$ is finite-dimensional. In particular, we may assume that $M$ is chosen so that $V_{\mathbf{m}} / M$ is simple.

Let $I \subseteq \mathfrak{n}$ be the annihilator of this quotient module. In [MZ2, Subsection 3.3] it is shown that any ideal in $\mathfrak{n}$ of finite codimension contains $1_{k}, 1_{k+1}, \ldots$ for some $k>0$. This implies that $\mathfrak{n} / I$ is nilpotent, $V_{\mathbf{m}} / M$ is 1 -dimensional, and $[\mathfrak{n}, \mathfrak{n}] \subseteq I$. Therefore, $l_{3}$ and $l_{4}$ act on $V_{\mathbf{m}} / M$ as zero, and thus $z\left(l_{3}-z^{2} 1_{1}\right)$ and $l_{4}-z^{3} 1_{1}$ act equally, which yields $z m_{3}=m_{4}$.

Assume now that $z m_{3}=m_{4}$ and consider the $\mathfrak{n}$-module $\mathbb{C}_{m_{2}, m_{3}}$ on which $\mathrm{l}_{1}$ acts via $-\frac{1}{z^{2}} m_{3}$ and $1_{2}$ acts via $m_{2}-\frac{1}{z} m_{3}$. Computing its restriction to $\mathfrak{a}_{z}$ one gets that the restriction is isomorphic to $\mathbb{C}_{\mathbf{m}}$. From the universal property of induced modules it follows that $V_{\mathbf{m}}$ surjects onto this $\mathfrak{n}$-module $\mathbb{C}_{m_{2}, m_{3}}$ and hence is reducible.

From the above proof it follows that in the case $z m_{3}=m_{4}$ the module $V_{\mathbf{m}}$ has a unique simple top isomorphic to the $\mathfrak{n}$-module $\mathbb{C}_{m_{2}, m_{3}}$ on which $1_{1}$ acts via $-\frac{1}{z^{2}} m_{3}$ and $I_{2}$ acts via $m_{2}-\frac{1}{z} m_{3}$. The induced module $\operatorname{Ind}_{z, \theta}\left(\mathbb{C}_{m_{2}, m_{3}}\right)$ is completely described in OW, MZ2].

For $k>4$ set

$$
m_{k}=-(k-4) m_{3} z^{i-3}+(k-3) m_{4} z^{i-4} .
$$

To simplify our notation, for $k \geq 2$ we will denote by $\hat{1}_{k}$ the element $1_{k}-z^{k-1} 1_{1} \in$ $\mathfrak{a}_{z}$. We will need the following property of $V_{\mathbf{m}}$ :

Lemma 5. Let $k \geq 2$. Then the module $V_{\mathbf{m}}$ has a basis $\left\{v_{n}^{(k)} \mid n \in \mathbb{N}_{0}\right\}$ such that $\hat{1}_{k} \cdot v_{n}^{(k)}=\left(m_{k}+n z^{k}(1-k)\right) v_{n}^{(k)}$.

Proof. Let $v$ be the canonical generator of $V_{\mathbf{m}}$. For $n \in \mathbb{N}_{0}$ denote by $V(n)$ the linear span of $v, 1_{1} v, \ldots, 1_{1}^{n} v$. We claim that $V(n)$ is invariant under the action of $\hat{1}_{k}$ and, moreover,

$$
\hat{\mathbf{1}}_{k} \cdot \mathfrak{I}_{1}^{n} v=\left(m_{k}+n z^{k}(1-k)\right) 1_{1}^{n} v \bmod V(n-1) .
$$

We show this by induction on $n$. If $n=0$, the claim is clear. To show the induction step, we compute (by moving $\hat{1}_{k}$ through one $l_{1}$ and using the inductive assumption in the first equality):

$$
\begin{aligned}
& \left(\mathrm{l}_{k}-z^{k-1} 1_{1}\right) \cdot 1_{1}^{n+1} v \\
& \quad=\left(m_{k}+n z^{k}(1-k)\right) 1_{1}^{n+1} v+\left[1_{k}-z^{k-1} 1_{1}, 1_{1}\right] 1_{1}^{n} v \bmod V(n) \\
& \quad=\left(m_{k}+(n+1) z^{k}(1-k)\right) 1_{1}^{n+1} v+(1-k)\left(1_{k+1}-z^{k} 1_{1}\right) 1_{1}^{n} v \bmod V(n) .
\end{aligned}
$$

This implies (3.2), and the statement of the lemma follows. 


\section{INDUCTION TO THE BOREL}

Denote by $\mathfrak{b}$ the standard Borel subalgebra of $\mathfrak{V}$, that is, the subalgebra generated by $\mathfrak{n}$ and $\imath_{0}$. Define $W_{\mathbf{m}}=\operatorname{Ind}_{\mathfrak{n}}^{\mathfrak{b}}\left(V_{\mathbf{m}}\right)$.

Proposition 6. If $z m_{3} \neq m_{4}$ and $\mathbf{m} \neq\left(m_{2}, 2 z m_{2}, 3 z^{2} m_{2}\right)$, then the $\mathfrak{b}$-module $W_{\mathbf{m}}$ is simple.

Proof. Consider $V=V_{\mathbf{m}}$ and let $W$ be the induced $\mathfrak{b}$-module. Then every element in $W$ can be written as $\sum_{i>0} I_{0}^{i} v_{i}$, where $v_{i} \in V$ and only finitely many of them are nonzero. Let $X$ be a nonzero submodule of $W$ and $x \in X$ be such that, when written in the above form, the maximal $i$ such that $v_{i} \neq 0$ is minimal possible; let it be $N$.

Since $V$ is a simple $\mathfrak{n}$-module, using the action of $\mathfrak{n}$ we may assume that $v_{N}$ is the canonical generator $v$ of $V$. We have $\hat{1}_{k} \cdot v=m_{k} v$.

We claim that $y=\left(\hat{\mathfrak{I}}_{k}-m_{k}\right) x$ is nonzero for some $k$ (which reduces $N$, giving a contradiction). For this we show that when we write $y$ in the above form, then the coefficient at $1_{0}^{N-1}$ will be nonzero. Clearly, $y$ will not contain any coefficient at $1_{0}^{N}$.

Look at $1_{0}^{N-1} V$ (modulo smaller powers of $1_{0}$ ) and choose there an eigenbasis for $\hat{1}_{k}$ as given by Lemma 5. There is one eigenvector $1_{0}^{N-1} v$ with eigenvalue $m_{k}$ and the spectrum of $\hat{I}_{k}$ simple, which means that this eigenvector is not in the image of $\hat{1}_{k}-m_{k}$. Hence it is enough to show that $y$ contains a nonzero component at this eigenvector coming from level $N$ (and hence this component cannot cancel with anything from level $N-1$, as it is not in the image).

Without loss of generality (namely, by factoring out smaller powers of $1_{0}$ ) we may assume $N=1$. In this case we get that the eigenvector at level 0 with eigenvalue $m_{k}+(1-k) z^{k}$ is $m_{k+1} v+z^{k} 1_{1} v$, while the contribution from level 1 is $k m_{k} v+(k-1) z^{k-1} l_{1} v$ (up to a nonzero constant). For these two vectors to be linearly dependent we get the equality $(k-1) m_{k+1} z^{k-1}=k m_{k} z^{k}$. This reduces, by recursion, to the equations $m_{k}=(k-1) m_{2} z^{k-1}$ for $k>2$. The claim follows.

We will also need the following property of $W_{\mathbf{m}}$ :

Lemma 7. Assume that $\mathbf{m}$ satisfies (1.1).

(a) The kernels of both $\hat{1}_{2}-m_{2}$ and $\hat{1}_{3}-m_{3}$ on $W_{\mathbf{m}}$ coincide with $\langle v\rangle$.

(b) The module $W_{\mathbf{m}}$ does not contain any element $x$ such that both $\left(\hat{1}_{2}-m_{2}\right) \cdot x=v$ and $\left(\hat{1}_{3}-m_{3}\right) \cdot x=z v$.

Proof. We start with claim (国). Set

$$
y_{2}:=\frac{1}{m_{3}-2 z m_{2}}\left(z 1_{0} v-1_{1} v\right)
$$

and

$$
y_{3}:=\frac{z}{2 m_{4}-3 z m_{3}}\left(z 1_{0} v-1_{1} v\right)
$$

and note that this is well-defined because of (1.1); moreover, $y_{2} \neq y_{3}$ (again by (1.1)). A direct computation shows that $\left(\hat{\mathfrak{I}}_{2}-m_{2}\right) \cdot y_{2}=v$ and $\left(\hat{\mathfrak{I}}_{3}-m_{3}\right) \cdot y_{3}=z v$.

Consider the filtration of $W_{\mathbf{m}}$ by $\mathbf{n}$-submodules given by the degree of $1_{0}$. Every layer of this filtration is isomorphic to $V_{\mathbf{m}}$, and from Lemma 5 it follows that the kernel of $\hat{1}_{2}-m_{2}$ on every layer of this filtration is 1-dimensional. The computation from the previous paragraph implies that each nonzero kernel element at the layer 
$k$ is sent to a nonzero kernel element at the layer $k-1$. This implies that the kernel of $\hat{1}_{2}-m_{2}$ on $W_{\mathbf{m}}$ coincides with the kernel of $\hat{1}_{2}-m_{2}$ on the first layer (corresponding to $1_{0}^{0}$ ). The latter one is exactly $\langle v\rangle$ by Lemma 5. This proves claim (国) for $\hat{1}_{2}-m_{2}$, and for $\hat{1}_{3}-m_{3}$ the arguments are similar.

From claim (a) and the computation above it follows that the equation $\left(\hat{1}_{2}-\right.$ $\left.m_{2}\right) \cdot x=v$ has solutions $y_{2}+\alpha v, \alpha \in \mathbb{C}$. None of these equals $y_{3}$ which implies claim (b), completing the proof.

\section{Proof of Theorem 1}

To prove Theorem 1 we will use a variation of the argument from the proof of Proposition 6] Assume that $\mathbf{m} \in \mathbb{C}^{3}$ satisfies (1.1). For $\theta \in \mathbb{C}$, consider the module $\operatorname{Ind}_{z, \theta}\left(\mathbb{C}_{\mathbf{m}}\right)$.

Denote by $\mathbf{M}$ the set of all infinite vectors $\mathbf{i}=\left(\ldots, i_{2}, i_{1}\right)$ with nonnegative integer coefficients in which only finitely many coordinates are nonzero. For $\mathbf{i} \in \mathbf{M}$ set

$$
\mathrm{I}^{\mathrm{i}}:=\ldots \mathrm{I}_{-2}^{i_{2}} \mathrm{I}_{-1}^{i_{1}} \in U(\mathfrak{V}) .
$$

For $\mathbf{i} \in \mathbf{M}$, the degree $\mathbf{d}(\mathbf{i})$ is defined as $\sum_{s>0} i_{s}$, and the weight $\mathbf{w}(\mathbf{i})$ is defined as $\sum_{s>0} s i_{s}$. Let $\mathbf{0}=(\ldots, 0,0,0)$, and for $s \in \mathbb{Z}_{0}$ let $\varepsilon_{s}$ be the element $\left(\ldots, i_{2}, i_{1}\right)$ such that $i_{s}=1$ and $i_{t}=0$ for all $t \neq s$. Define a total order $\prec$ on $\mathbf{M}$ recursively as follows: $\mathbf{i} \prec \mathbf{j}$ if and only if

- $\mathbf{w}(\mathbf{i})<\mathbf{w}(\mathbf{j})$; or

- $\mathbf{w}(\mathbf{i})=\mathbf{w}(\mathbf{j})$ and $\mathbf{d}(\mathbf{i})<\mathbf{d}(\mathbf{j}) ;$ or

- $\mathbf{w}(\mathbf{i})=\mathbf{w}(\mathbf{j})$ and $\mathbf{d}(\mathbf{i})=\mathbf{d}(\mathbf{j})$ and

$$
\min \left\{s \mid i_{s} \neq 0\right\}>\min \left\{s \mid j_{s} \neq 0\right\} \text {; or }
$$

- $\mathbf{w}(\mathbf{i})=\mathbf{w}(\mathbf{j})$ and $\mathbf{d}(\mathbf{i})=\mathbf{d}(\mathbf{j})$ and

$$
\min \left\{s \mid i_{s} \neq 0\right\}=\min \left\{s \mid j_{s} \neq 0\right\}=p
$$

and $\mathbf{i}-\varepsilon_{p} \prec \mathbf{j}-\varepsilon_{p}$.

Clearly, the element $\mathbf{0}$ is the minimum element with respect to this order.

Every element in $\operatorname{Ind}_{z, \theta}\left(\mathbb{C}_{\mathbf{m}}\right)$ can be uniquely written as a sum of $1^{\mathbf{i}} v_{\mathbf{i}}$, where $\mathbf{i} \in \mathbf{M}$ and $v_{\mathbf{i}} \in W_{\mathbf{m}}$ (where only finitely many of the $v_{\mathbf{i}}$ 's are nonzero). If $x$ is a nonzero element written in this form, then the support of $x$ is defined as the following finite set:

$$
\operatorname{supp}(x):=\left\{\mathbf{i}: v_{\mathbf{i}} \neq 0\right\}
$$

and the maximal term $\mathbf{t}(x)$ of $x$ is the maximal element in $\operatorname{supp}(x)$ (with respect to $\prec)$. Let $M$ be a nonzero submodule in $\operatorname{Ind}_{z, \theta}\left(\mathbb{C}_{\mathbf{m}}\right)$. Denote by $\mathbf{i}$ the minimal element in the set $\{\mathbf{t}(x): x \in M, x \neq 0\}$. To prove Theorem 1 we have to show that $\mathbf{i}=\mathbf{0}$.

Lemma 8. Let $x \in M, x \neq 0$, and $u \in \mathfrak{b}$. If $u x \neq 0$, then $\mathbf{t}(u x) \preceq \mathbf{t}(x)$.

Proof. By linearity, it is enough to prove the claim for $u=1_{k}$ and $x=1^{\mathbf{i}} v_{\mathbf{i}}$. We have $1_{k} \cdot 1^{\mathbf{i}} v_{\mathbf{i}}=1^{\mathbf{i}} 1_{k} \cdot v_{\mathbf{i}}+\left[1_{k}, 1^{\mathbf{i}}\right] v_{\mathbf{i}}$. From the definition of $\prec$ it follows that, moving $l_{k}$ to the right in $\left[1_{k}, 1^{\mathbf{i}}\right]$, we get a linear combination of some $\mathbf{1}^{\mathbf{j}}$ (with possible element from $\mathfrak{b}$ on the right) where $\mathbf{j} \preceq \mathbf{i}$. The claim follows. 
Assume that $\mathbf{i} \neq \mathbf{0}$ and let $x \in M$ be some nonzero element with maximal term i. Since $W_{\mathbf{m}}$ is a simple module by Proposition 6 , without loss of generality we may assume $v_{\mathbf{i}}=v$ (the canonical generator of $V_{\mathbf{m}}$ ). For $k \geq 2$ consider the element $y_{k}:=\left(\hat{\mathfrak{l}}_{k}-m_{k}\right) \cdot x$, and we have either $y_{k}=0$ or $\mathbf{i} \notin \operatorname{supp}\left(y_{k}\right)$. Therefore $y_{k} \neq 0$ implies $\mathbf{t}\left(y_{k}\right) \prec \mathbf{i}$ by Lemma 8 , which gives a contradiction completing the proof of Theorem 1. In what follows we show that either $y_{2} \neq 0$ or $y_{3} \neq 0$.

Let $p:=\min \left\{s: i_{s} \neq 0\right\}$ and consider first the case $p>1$. We claim that $\mathbf{j}:=\mathbf{i}-\varepsilon_{p}+\varepsilon_{p-1}$ belongs to either $\operatorname{supp}\left(y_{2}\right)$ or $\operatorname{supp}\left(y_{3}\right)$, which implies that at least one of these elements is nonzero. Assume that this is not the case and let $k \in\{2,3\}$. Let $\mathbf{i}^{\prime} \in \operatorname{supp}(x)$ be different from $\mathbf{i}$. Using the definition of $\prec$, it is easy to see that, writing $\left[\hat{\mathfrak{l}}_{k}-m_{k}, \mathrm{I}^{\mathbf{i}^{\prime}}\right]$ as a linear combination of $\beth^{\mathbf{s}} u_{\mathbf{s}}$, where $u_{\mathbf{s}} \in \mathfrak{b}$, all $\mathbf{s}$ which will appear with nonzero $u_{\mathbf{s}}$ satisfy $\mathbf{s} \prec \mathbf{j}$.

At the same time, $\left[\hat{\mathfrak{l}}_{k}-m_{k}, \mathbf{l}^{\mathbf{i}}\right]$ contributes with $-i_{p}(p+1) z^{k-1} \mathbf{l}^{\mathbf{j}}$. If $y_{k}=0$, then to cancel this contribution, we thus must have $\mathbf{j} \in \operatorname{supp}(x)$ and $v_{\mathbf{j}} \in W_{\mathbf{m}}$ should satisfy $\left(\hat{\mathbf{1}}_{k}-m_{k}\right) \cdot v_{\mathbf{j}}=i_{p}(p+1) z^{k-1} v$. However, such $v_{\mathbf{j}}$ does not exist by Lemma 7(b), which completes the proof in the case $p>1$.

Assume now that $p=1$. Our argument will be similar to the one we used above, however, it will require a computationally more complicated analogue of Lemma 7 . We claim that $\mathbf{j}:=\mathbf{i}-\varepsilon_{1}$ belongs to either $\operatorname{supp}\left(y_{2}\right)$ or $\operatorname{supp}\left(y_{3}\right)$. Assume that this is not the case. Consider the coefficient at $1^{\mathbf{j}}$ in both $y_{2}$ and $y_{3}$. Similarly to the above, the only contribution to this coefficient comes from $\left[\hat{1}_{k}-m_{k}, I^{\mathbf{i}}\right]$ and from $\left(\hat{\mathfrak{l}}_{k}-m_{k}\right) \cdot v_{\mathbf{j}}$. So, if this coefficient is zero, these two contributions should cancel each other for both $k=2$ and $k=3$.

One checks that the contribution from $\left[\hat{\mathfrak{l}}_{2}-m_{2}, \mathrm{I}^{\mathrm{i}}\right]$ equals exactly

$$
i_{1}\left(-31_{1}+2 z 1_{0}+z\left(i_{1}-1\right)\right) v
$$

and the contribution from $\left[\hat{\mathfrak{I}}_{3}-m_{3}, \mathbf{l}^{\mathbf{i}}\right]$ equals

$$
i_{1}\left(-4 m_{2}-4 z 1_{1}+2 z^{2} \beth_{0}+z^{2}\left(i_{1}-1\right)\right) v .
$$

Now the claim follows, similarly to the above, from the following lemma:

Lemma 9. Let $t \in \mathbb{N}$. If $\mathbf{m}$ satisfies (1.1), then $W_{\mathbf{m}}$ does not contain any element $x$ such that

$$
\left\{\begin{array}{l}
\left(\hat{\mathrm{I}}_{2}-m_{2}\right) \cdot x=t\left(-3 \mathrm{l}_{1}+2 z \mathrm{l}_{0}+z(t-1)\right) v \\
\left(\hat{\mathrm{I}}_{3}-m_{3}\right) \cdot x=t\left(-4 m_{2}-4 z \mathrm{l}_{1}+2 z^{2} \mathfrak{l}_{0}+z^{2}(t-1)\right) v .
\end{array}\right.
$$

Proof. By Lemmal7(a), each of the above equations, if solvable, has a 1-dimensional set of solutions (which differ by scalar multiples of $v$ ). It is straightforward to check that the element

$$
\begin{aligned}
\frac{t}{2 z^{2} m_{2}-z m_{3}}( & -z^{3} 1_{0}^{2} \\
& -\frac{2 z^{5}(1+t) m_{2}-z^{4}(4+t) m_{3}+2 z^{2} m_{2} m_{3}+2 z^{3} m_{4}-z m_{3}^{2}}{2 z^{2} m_{2}-z m_{3}} 1_{0} \\
& +2 z^{2} 1_{0} 1_{1} \\
& \left.+\frac{2 z^{4} t m_{2}+4 z^{2} m_{2}^{2}-z^{3}(3+t) m_{3}-2 z m_{2} m_{3}+2 z^{2} m_{4}}{2 z^{2} m_{2}-z m_{3}} 1_{1}-z 1_{1}^{2}\right) \cdot v
\end{aligned}
$$


solves the first equation and that the element

$$
\begin{aligned}
\frac{t}{3 z m_{3}-2 m_{4}}\left(-z^{3} 1_{0}^{2}+\left(4 z m_{2}-\frac{2}{z} m_{4}-t z^{3}\right)\right. & 1_{0}+2 z^{2} 1_{0} 1_{1} \\
& \left.+\left(t z^{2}-z^{2}-4 m_{2}+\frac{3}{z} m_{3}\right) 1_{1}-z 1_{1}^{2}\right) \cdot v
\end{aligned}
$$

solves the second equation. Comparing the coefficient at $1_{0}^{2}$, from (1.1) it follows that these two elements are different; moreover, they do not differ by a scalar multiple of $v$. The claim of the lemma follows.

Both solutions in the proof of Lemma 9 were found and also Gröbner basis computations in the proof of Proposition 3 were performed using MAPLE, but it is straightforward to check that they are correct. Note that from the definition of our modules it follows immediately that different values of parameters give rise to nonisomorphic simple modules. It is also easy to check (looking at the action of $\mathfrak{a}_{z}$ ) that our modules are not isomorphic to any of the previously known simple Virasoro modules (confer [LZ, Theorem 17]).

\section{ACKNowledGements}

The major part of this work was done during the visit of the second author to Uppsala in May 2012. The hospitality and financial support of Uppsala University are gratefully acknowledged. The first author is partially supported by the Royal Swedish Academy of Sciences and the Swedish Research Council.

\section{REFERENCES}

[BM] Punita Batra and Volodymyr Mazorchuk, Blocks and modules for Whittaker pairs, J. Pure Appl. Algebra 215 (2011), no. 7, 1552-1568, DOI 10.1016/j.jpaa.2010.09.010. MR2771629 (2012c:17013)

[CM] Charles H. Conley and Christiane Martin, A family of irreducible representations of the Witt Lie algebra with infinite-dimensional weight spaces, Compositio Math. 128 (2001), no. 2, 153-175, DOI 10.1023/A:1017566220585. MR1850181(2002f:17041)

[FF] B. L. Fer̆gin and D. B. Fuks, Verma modules over a Virasoro algebra, Funktsional. Anal. i Prilozhen. 17 (1983), no. 3, 91-92. MR714236 (84i:17009)

[GLZ] Xiangqian Guo, Rencai Lu, and Kaiming Zhao, Fraction representations and highestweight-like representations of the Virasoro algebra, J. Algebra 387 (2013), 68-86, DOI 10.1016/j.jalgebra.2013.04.012. MR.3056686

[IK] Kenji Iohara and Yoshiyuki Koga, Representation theory of the Virasoro algebra, Springer Monographs in Mathematics, Springer-Verlag London, Ltd., London, 2011. MR2744610 (2011m:17058)

[KR] V. G. Kac and A. K. Raina, Bombay lectures on highest weight representations of infinitedimensional Lie algebras, Advanced Series in Mathematical Physics, vol. 2, World Scientific Publishing Co., Inc., Teaneck, NJ, 1987. MR1021978 (90k:17013)

[LZ] R. Lü, K. Zhao; Irreducible Virasoro modules from irreducible Weyl modules. J. Algebra, to appear.

[LGZ] Rencai Lü, Xiangqian Guo, and Kaiming Zhao, Irreducible modules over the Virasoro algebra, Doc. Math. 16 (2011), 709-721. MR2861395

[Mt] Olivier Mathieu, Classification of Harish-Chandra modules over the Virasoro Lie algebra, Invent. Math. 107 (1992), no. 2, 225-234, DOI 10.1007/BF01231888. MR.1144422 (93d:17034)

[MZ1] Volodymyr Mazorchuk and Kaiming Zhao, Classification of simple weight Virasoro modules with a finite-dimensional weight space, J. Algebra 307 (2007), no. 1, 209-214, DOI 10.1016/j.jalgebra.2006.05.007. MR2278050 (2007h:17028) 
[MZ2] V. Mazorchuk, K. Zhao; Simple Virasoro modules which are locally finite over a positive part. Selecta Math. (N.S.), to appear.

[OW] Matthew Ondrus and Emilie Wiesner, Whittaker modules for the Virasoro algebra, J. Algebra Appl. 8 (2009), no. 3, 363-377, DOI 10.1142/S0219498809003370. MR2535995 (2010f:17040)

[Zh] Hechun Zhang, A class of representations over the Virasoro algebra, J. Algebra 190 (1997), no. 1, 1-10, DOI 10.1006/jabr.1996.6565. MR1442144(98c:17025)

Department of Mathematics, Uppsala University, Box 480, SE-751 06, Uppsala, SWEDEN

E-mail address: mazor@math.uu.se

Department of Mathematics, Williams Hall, Ithaca College, Ithaca, New York 14850

E-mail address: ewiesner@ithaca.edu 